\title{
Die Blätterschicht der electrischen Organe von Raja in ihren genetischen Beziehungen zur quergestreiften Muskelsubstanz.
}

Von

Th. W. Engeĩmann.

Hierzu Tafel II.

Einleitung.

Durch A. B a bu ch ins ${ }^{1}$ ) denkwürdige Entdeckung der Entwickelung der electrischen Organe ans Maskelfasern ist, wie durch jeden grossen anatomischen Fund, der Physiologie eine Fülle neuer Probleme, zugleich aber auch die Aussicht auf Lösung wichtiger alter Aufgaben eröffnet worden. Eine genaue Verfolgung der Processe, durch welche sich die zuckende Faser zum electrischen Apparat umbildet, musste vor Allem Aufschluss versprechen über Sitz und Wesen sowohl einerseits des Contractionsvermögens als andererseits der electromotorischen Wirksamkeit. Es war zu erwarten, dass in dem Maasse, als das Zuckungsvermögen sich zurückbildete, auch die materiellen Grundlagen, an welche dasselbe gebunden ist, einer Rückbildung unterliegen würden, und dass auf der anderen Seite mit zunehmender Steigerung der electromotorischen Fähigkeiten die Organisationsverhältnisse, welche Träger dieser letzteren Fähigkeiten sind, sich deutlicher und deutlicher ausbilden würden.

Nirgends in der organischen Natur ist ein ähnlich grossartiger, jene fundamentalen Erscheinungen vitaler Erzeugung mecha-

1) Babuchin, Entwickelung der electrischen Organe und Bedeutung der motorischen Endplatten. Vorläufige Mittheilung. Centralbl. f. d. med. Wissensch. 1870. No. 16. 17. (Die Resultate sind zuerst auf der zweiten russischen Naturforscher-Versammlung am 25. Aug. 1869 mitgetheilt worden.) - Ueber die Bẹdeutung und Entwickelung der pseudoelectrischen Organe. Ebenda 1872. No. 35 . 
nischer und electrischer Energie betreffender Structur- und Functionswechsel in auch nur annähernd vollkommener Weise der Untersuchung zugänglich, ja ïberhanpt bisher wahrgenommen. Doch haben gerade die angedeuteten Probleme bisher noch nicht die gewünschte allseitig eingehende Behandlung seitens der zahlreichen vortrefflichen Forscher gefunden, welche die Endeckung des russischen Histiologen weiter verfolgten. Im Grossen und Ganzen ist man nicht viel weiter gekommen, als za dem schon von Babuch in formulirten allgemeinen Ergebniss, dass die Substanz der Muskelfaser unter Verlust der Contractilität und des specifischen Baues zu dem einen, dem sogenannten muskulären oder metasarkoblastischen, die motorische Nervenendigung zu dem anderen, dem nervösen Gliede des electrischen Organs sich umbildet. Die Einzelheiten dieser Umbildungsvorgänge sind, wie gleichfalls schon B a b a ch in betont bat ${ }^{1}$, je nach der Thierart ausserordentlich verschieden. Bei den einen Arten (Torpedo and Verwandte) hebt die Umwandlung schon an, ehe noch die jungen Zellen deutliche Querstreifen oder sichere Zeichen von Contractilität besitzen und lässt auch das entwickelte, relativ einfach gebaute Organ in seiner histiologischen Structur keine Andeutung einer genetischen Beziehung zu Muskelfasern mehr erkennen. Bei anderen Arten (R a j a) bilden sich zunächst histiologisch vollständig differenzirte, functionsfähige quergestreifte Muskelfasern aus und diese wandeln sich nachträglich in äusserst complicirte Organe um, welche noch auf der Höhe ihrer Ausbildung, in dem Bau der lamellösen, oft auch der sogenannten alveolären Schicht deutliche Spuren ihrer Abstammung von quergestreiften Elementen tragen. Ja auch innerbalb desselben engeren Formenkreises zeigen sich, wie E wa $\mathrm{rt}^{2}$ ) für Raja in einer vortrefflichen Untersuchungsreihe nachwies, viel-

1) Vgl. besonders Babuchin, Uebersicht der neuen Untersuchungen über Entwickelung, Bau and physiologische Verhältnisse der electrischen und pseudoelectrischen Organe. Arch. für Anat, Physiologie u. s. w. von Reichert und du Bois-Reymond 1876. p. 540-541.

2) J. C. Ewart, The electric organ of the skate. On the Development of the Electric Organ of Raja batis. Philos. Transact. London. Vol. 179. 1888. p. 399. Pl, 66, 67. - On the Structure of the Electr. Organ of Raja circularis. Ibid. p. 410. P1. 68. - The Electric Organ of Raja radiata. Ibid. p. 539. Pl. 79. 80 . 
fache phylogenetisch sehr lehrreiche Modificationen im Auftreten, Verlauf and Endresultat jenes Umwandlungsproeesses.

Es ist zu erwarten, dass Fälle wie die letzteren die zahlreichsten und werthvollsten Aufschliusse bieten werden, denn hier, bei $R a j a$, ist es ein morphologisch und physiologiseh nachweislich hoch differenzirter Apparat, welcher die histiologische und functionelle Umgestaltung erfährt, nicht wie bei Tor ped o eine indifferente, nichts Specifisches zeigende Masse, deren Organisation wesentlich jenseits des mikroskopischen Unterscheidungsvermögens liegt. Freilich erreicht in den letzteren Fällen die electrische Leistungsfähigkeit und damit jedenfalls die dieser zu Grunde liegende specifische materielle Ausbildung eine unvergleichlich höbere Stufe. "Was ein guter Haken werden will, muss früh sich biegen," bemerkt mit Recht $n d u$ B o i s Re y m o n d"1). Beruht hierauf doch die frühere Unterscheidung "electrischer" von "pseudoelectrischen" Organen. Aber auch in diesem Umstand liegt doch insofern ein Vortheil, als er der vergleichend histio-physiologischen Betrachtung, die neben der genetischen ohnehin nicht entbebrt werden kann, neue Handhaben gewährt.

Von grossem heuristischen Werth ist ferner bei $R$ aja der schon von $\mathrm{Babuchin}$ und namentlich von $\mathrm{Ewart}$ bemerkte, auch von $\mathrm{M} \mathrm{u} \mathrm{s} \mathrm{k} \mathrm{e} \mathrm{s}^{2}$ ) betonte Umstand zu erachten, dass die Metamorphose relativ langsam und nicht bei allen Elementen desselben Thiers gleichzeitig erfolgt, und dass sie anch im Allgemeinen nicht an eine eng begrenzte Phase der embryonalen Entwickelung der Thiere gebunden $z \mathfrak{u}$ sein scheint, sondern sich noch weit ins postembryonale Leben hinein fortsetzt.

Endlich gehören die Arten der Gattung Raja zu den allerverbreitetsten, überall und allezeit in grosser Menge zu beschaffenden Seefischen.

Die vorliegende histiologische Untersuchung setzt sich nur zum Ziel den Nachweis der näheren genetischen Beziehungen zwischen den Querstreifen der Muskelfasern und den Lamellen der sogenannten mäandrischen oder gestreiften Sehicht, welche bei den

1) E. du Bois-Reymond, Dr. Carl Sachs Untersuchungen am Zitteraal. Leipzig 1881. p. 62.

2) L. J. J. M uskens', Zur Kenntniss der elektrischen Organe. Tydschr. der Nederl. Dierkund. Vereeniging 2. R. D. IV. 1892. 
meisten Rajaspecies, unter der Nervenendplatte gelegen, einenHauptbestandtheil der electrischen Organe im Schwanze dieser Fische bildet. Die bisherigen Untersuchungen lehren nar soviel mit Gewissheit, $d$ a s s solche genetischen Beziehungen bestehen. B a bue h in ${ }^{1}$ ) entdeckte, dass die mäandrische Schicht direct aus der quergestreiften Substanz hervorgeht und $\mathrm{E} w$ a $\mathrm{rt}^{2}$ ) zeigte, dass sie bei $\mathrm{Raja}$ radiata auch im entwickelten Thier noch ganz wie gewöhnliche quergestreifte Muskelsubstanz aussieht, und dass Uebergänge von dieser phylogenetisch niedrigsten zu den durch complicirten mäandrischen Verlauf ausgezeichneten höheren Formen (z. B. Raja circularis, batis) vorkommen. Die wichtige Frage, welche Homologien zwischen den Lamellen der ausgebildeten Blätterschicht und den Querschichten der Muskelfasern bestehen, ist noch unentschieden. Einige Abbildungen und Bemerkungen von $\left.\mathrm{B} a b \mathfrak{a b i n}{ }^{3}\right)$ können den Glauben erwecken, als ob. die stark lichtbrechenden dünnen Blätter der Lamellenschicht des entwickelten Organs den doppelbrechenden Querscheiben, die zwischen: liegenden scbwach lichtbrechenden dicken Schichten den isotropen Scheiben der Muskelfasern entsprechen. Die Abbildungen von Ew a t $^{4}$ ) andererseits erwecken den Schein, "als ob das Gegentheil der Fall sei. Doch fehlen auch hier nähere Angaben und ist speciell das Verhalten im polarisirten Licht nicht geprüft. Andere Daten, welcbe etwa Sicherheit versehaffen könnten, habe jeb in der. Literatur nicht ausfindig machen können.

Uyd doch ist, wie ich glaube, die Entscheidung gerade dieser besonderen Fragen von Bedentung.

Die neueren Untersuchungen über Muskelstructur haben einen ausserordentlich complicirten, aber bei allen quergestreiften Muskeln aller Thiere wesentlich gleichen Bau der contractilen Fibrillen aufgedeckt. Man hat, wie ich gezeigt habe ${ }^{5}$ ), allen Grund anzu-

1) Centralbl. f. d. med. Wiss. 1872: No. 35.

2) a. a. O. p. 545. Pl. 80. Fig. 12A.

3) Centralbl. f. d. med. Wiss. 1872. No. 35. p. 546 u. 547. - Archiv f. Anat. Physiol. u. s; w. 1876, p. 539. Taf. XII. Fig. 19 n. 20.

4) a. a. 0. Pl. 66. Fig. 2, 3. Pl. 79. Fig. 4 und 5. Pl. 80. Fig. 8.

5) Th. W. Engelmann, Mikroskopische Untersuchungen über die quergestreifte Muskelsubstanz. Pfïüger's Archiv. 7. Bd. 1873. p. 162 u. flg: - Onderzoek. physiol. lab. Utrecht, (3) II. 1873. p. 204 u. flg. - Ueber den Ursprung der Muskelkraft. Leipzig 1893. Zweite Aufl, p. 11 u. flg. p. 44 u. flg. 
nehmen, dass dieser morphologisehen Differenzirung eine physiologische Arbeitstheilung in dem Sinne entspricht, dass die doppelbrechenden Fibrillenglieder, und zwar speciell die von $\mathrm{R}$ oll et neuerdings als metabole bezeichneten Theile, Sitz der verkürzenden Kräfte sind, die von Rollett als a rimetabole unterschiedenen, in der Hauptsache isotropen Glieder dagegen wesentlich bei den Leitungsvorgängen und damit auch wesentlich bei den electromotorischen Processen betheiligt sind. Es musste daher eine der ersten Aufgaben sein, genau festzuistellen, welche Aenderungen jede dieser beiden Schichten bei der Umwandlung der Querstreifen in "die Querbänder" der Lamellenschicht des electrischen Organs erleidet.

Ich selbst war anfangs, wegen der sehr geringen Breite der Querstreifung embryonaler Vertebratenmuskeln, zweifelhaft, ob sich die Frage mit unseren optischen Hilfsmitteln wirklich mit völliger Sicherheit würde beantworten lassen. Unwillkürlich kam das Bedauern auf, zu dem übrigens auch jetzt noch Grund besteht, dass nicht auch bei Arthropodenmuskeln eine Verwandlung in electrische Organe vorkommt. Inzwischen widerlegte eine nähere Prüfung mit starken guten Objectiven jene Zweifel und fihrte zur Ermittelung der im Folgenden za beschreibenden, unschwer zu bestätigenden Thatsachen, welche, wie ich glaube, die endgiltige Antwort enthalten.

Unsere Untersuchungen erstrecken sich hauptsächlich auf Raja elavata, von denen wir durch gutige Vermittelung von Dr. Hoek im Helder Embryonen verschiedenen Alters erhielten. Herr Cand.med Muskens, durch eigene Studien mit der Morphologie und Genese der electrischen und pseudoelectrischen Organe bereits vertraut ${ }^{1}$ ), verpflichtete mich durch Anfertigung yon Schnittserien durch die, meist in Pikrinschwefelsäure erbärteten Organe und unterstützte mich überhaupt bei Beschaffung, Herstellung und Untersuchung: des histiologischen Materials auf das Eifrigste. Ausserdem bin ich Herrn Professor J. C. E w a r t in Edinburgh zu Dank verpflichtet für Uebersendung einer Reihe mikroskopischer Präparate, welche verschiedene Entwickelungsstufen der Organe von $R$ aj a radiata und Schnitte durch das entwickelte Organ von Raja circularis

1) Vgl. L. J. J. Muskens, Zur Kenntniss der electrisehen Organe. a. a. 0 . 
enthalten. Die Vergleichung dieser Objecte, namentlich derer von R. radiata, war besonders erwünscht, weil dieselben nach Ewarts Entdeckung sich viel weniger weit vom Muskelfasertypus entfernen als die von $R$. batis und clavata.

\section{Ergebnisse der histiologischen Untersuchung.}

1. Die dü nnen, stark lichtbrechenden Lamellen des electriscben Organessind denarimetabolen ${ }^{1}$ ) (isotropen), die dicken schwach lichtbrechenden den metabolen') (anisotropen) Schichtender quergestreiften Muskelfasern homolog.

Den Beweis dieser Homologie ermöglicht die Thatsache, dass alle Uebergangsstufen vom typischen Bild der quergestreiften Muskelsnbstanz zum typischen Bild der mäandrischen Schicht des entwickelten electrischen Organs sich nachweisen lassen.

Fig. 1-5 zeigt die Entwickelung des Organs von $\mathrm{Raj}$ a c lavata von der Faser- bis zur Kästchenform nach in Pikrinsch wefelsäure erhärteten, in Balsam eingebetteten Präparaten. Alle stammen von einem etwa $7 \frac{1}{2} \mathrm{~cm}$ langen Embryo, dessen Schwanz in eine Serie ron Frontalschnitten von etwa $0,01-0,02 \mathrm{~mm}$ Dicke zerlegt war. Die jüngsten Zustände liegen im hintersten Abschnitt des Schwanzes. Viele Schnitte zeigten sämmtliche in Fig. 1-5 abgebildete Entwickelungsstufen in mehrfachen Exemplaren und mit mannichfachen Uebergängen.

Eine genaue vergleichende Betrachtung der Figuren lässt zunächst keinen $Z_{w}$ eifel, dass die dunklen schmalen Querstreifen der Endanschwellung der Muskelfaser Fig. 1 den dünnen dunklen Querbändern im Kästchen Fig. 5, die hellen breiten Querbänder von Fig. 1 den hellen breiten Bändern von Fig. 5 entsprechen. Die Figg. 2, 3, 4 vermitteln den Uebergang zwischen Fig. 1 und 5 in ganz continuirlicher Weise. Aber auch innerhalb der einzelnen Fasern Fig. 1, 2, 3, ja auch noch innerhalb Fig. 4 ist der allmäh-

1) Ich folge in der Bezeichnung der verschiedenen Schichten der quergestreiften Substanz der von Al. Rollett (Denkschriften der math. naturw. Classe der kais. Akad. d. Wiss. Bd. LVIII. p. 67. 1891.) vorgeschlagenen Terminologie. 
liche Uebergang der Muskelquerstreifen in die Lamellenstreifung zu sehen.

Zur sicheren Feststellung bedarf es starker Vergrösserung, denn die Breite der Querstreifen in den jungen Muskelfasern ist wie bei allen Vertebratenmuskeln sehr gering. Eine gute 500 malige Vergrösserung genügt zwar, wie Figg. 1-5 zeigen, ist aber nicht bequem. Es sind deshalb einzelne Stellen der verschiedenen Objecte noch besonders bei einer etwa 1500 maligen Vergrösserung (Zeiss' apochrom. Syst., $3 \mathrm{~mm}, 1,4 \mathrm{~mm}$ Apert., Comp. Ocul. 12) abgebildet und zwar je zur Hälfte so, wie sie im gewöhnlichen Lichte oder zwischen parallelen Nicols, zur Hälfte so, wie sie bei gekreuzten Nicols erscheinen, wenn die Längsaxe der Fasern unter $45^{\circ}$ gegen die Schwingungsebenen der Polarisationsvorrichtung orientirt ist.

Fig. 1 a zeigt ein Stück der Querstreifung. der Muskelfaser Fig. 1 aus dem mittleren Drittel ihres faserförmigen Theils, Fig. 1b ein solches aus der Mitte der Endanschwellung, Fig. 2 a aus der Mitte der Endanschwellung der Faser Fig. 2, Fig. 3a, 4a, 5a entsprechende Partien aus Fig. 3-5, Fig. 6 einige Lamellenstuicke aus einem völlig ausgebildeten Kästchen des erwachsenen Thiers. In Fig. 1e ist zum Vergleich das Bild der Querstreifung einer benachbarten, nicht zur Umwandlung in ein electrisches Kästchen bestimmten Schwanzmuskelfaser desselben Embryos gegeben. Ein Zweifel rücksichtlich der Homologien kann angesichts dieser Figuren nicht bestehen. Auf die im Einzelnen bernerkbaren Unterschiede wird unten näher eingegangen werden.

Bei Raja radiata ist die Blätterschicht an den uns vorliegenden, in Sublimat erbärteten und in Balsam eingebetteten Präparaten Ewarts im entwickelten oder nahezu entwickelten Zustand ${ }^{1}$ ) ausserordentlich eng, im allgemeinen der proximalen Endfläche des Organs parallel quergestreift. Die Streifung ist durchschnittlich wohl 2 bis 3 mal enger als in unserer Fig. 1, was natürlich die Untersuchung bedeutend erschwert. Doch zeigen gute Immersionssysteme, ja auch schon die stärksten Trockenlinsen (Zeiss' F mit Correction z. B.) ähnlich wie bei Muskelfasern im Stadium der Umkehrung, schmale dunkle Streifen durch breitere, hellere

1) Das eine der Präparate stammt von einem Exemplar von " 10 inches" Schwanzlänge, ein anderes von einem Thier mit "71/2 inches" langen Schwanz, 
Bänder getrennt. Verfolgt.man die Streifung in distaler Richtung nach dem faserförmigen Stiel des Organs zu, so wird sie meist rasch breiter, obne übrigens ihren Charakter wesentlich zu ändern. Sie zeigt auch da das Bild des Umkehrungsstadiums oder allenfalls des Uebergangsstadiums.

Dasselbe sieht man in den jüngeren, Ewarts Pl. 79 Fig. 5 und 6 entsprechenden keulenförmigen Zuständen, welche durchgängig bis an das proximale Ende hinan wesentlich breiter gestreift sind als die lamellöse Schicht des entwickelten Organs. Doch wird schon in Stadien, welche etwa Ewarts Fig. 8 und 9 gleichen, die Querstreifung am proximalen Ende zuweilen merklich enger.

Die Homologie der schmalen dunklen Querstreifen der keulenförmigen Anschwellung mit der arimetabolen Schicht des cylindrischen Theils der Faser ist bei R. radiata durch schrittweises Verfolgen der in derselben Faser in distaler Richtung aufeinander folgenden Schichten ebenso sicher wie bei Raja clavata (Fig. 1 -3) zu verfolgen.

Auch die in Fig. 7 von uns abgebildeten, von einem nicht näher bestimmten Rajaembryo vom Helder herrïhrenden Objecte ergeben das gleiche Resultat. Das in Fig. $7 \mathrm{~b}$ bei 1500 maliger Vergrösserung gezeichnete Bild gilt für alle Stellen der Fasern II-VI, gleichviel ob sie im schmalen oder im keulenförmig verdickten Theil der Faser liegen. Hier hat man sogar noch das typische Bild der ruhenden, oder doch noch im "Anfangsstadium" befindlichen Muskelsubstanz: helle, durch eine schmale dunkle Linie $z$ balbirte Bänder (arimetabole oder isotrope Schicht) nnd dunklere, nur wenig breitere, durch ein schmales belleres Band $(h$ halbirte Querbänder (metabole Schicht).

2. Bei der 0 ntogenese der mäandrischen Schicht durchläuft keineswegs jedeLamelledieganze phylogenetische Stufenreihe, sondern es werden innerbalb gewisser Grenzen die friberen Phasen um so vollständiger und weiter übersprungen, je später im La ife der Entwickelung der einzeluen Faser zum electrischen Kästchen di e Lamelle angelegtwird.

Wenn man Bilder wie Fig. 1, 2, 3, 7 betrachtet, könnte man zunächst versucht sein zu meinen, dass die Umwandlung der Querstreifen der Maskelfaser in die Lamellen des electrischen Or- 
gans innèrhalb jeder Faser in der Richtung nacli dem proximalen Faserende hin fortschreite, derart, dass die weiter distal gelegenen Querschichten die jüngeren seien und sich, je weiter vom proximalen Ende entfernt; um so später in Lamellen umzubilden anfingen. Eine nähere Zergliederung lehrt jedoch, dass in Wirklichkeit das Umgekehrte statt hat. Es erfolgt nämlich an dem proximalen Faserende, während dasselbe zum electrischen Organ anschwillt, eine fortwährende Anfiugung never Schichten, der Art, dass jede neueste, terminale Sehicht gleich beim ersten Entstehen den definitiven Lamellen des Kästchens ähnlicher angelegt wird, als die zuvor gebildeten, in distaler Richtung auf sie folgenden.

Eine Vermehrung der Zahl der Schichten, durch Vermehrung der Zahl der Muskelfächer, kommt bei den Fasern von R. elavata zweifellos, wenigstens anfangs längere Zeit vor. Denn die.Länge der Fasern wächst, wie Vergleichung der verschiedenen Entwickelungszustände lehrt, viel schneller als die Höhe der Muskelfächer. Die Vermehrung der Schichten kann aber wobl nur durch Anlagerung an den Enden, nicht durch Einschaltung neuer Glieder in den Verlauf bereits gebildeter Muskelfibrillen stattfinden. Ich habe wenigstens nie Bilder finden können, die für solehe Einsebaltung sprächen. Die Fächer sind bei R. clavata durch die ganze Länge der jungen Fasern merklich gleich hoch und gleich gebaut.

Dass die Anfugung an beiden Enden der Faser geschiebt, ist darum wahrscheinlich, weil sich an beiden Enden oder in ihrer Näbe besondere Anhäufungen von Kernen und Protoplasma finden. Am proximalen Ende erreicht aber diese Anhäufung sebr bald einen viel höheren Grad als am terminalen, die Neubildung wird deshalb auch hier wohl in höherem Grade stattfinden.

Wie lange diese Neubildung hier erfolgt, wird für jede Rajaspecies durch besondere Untersuchungen zu ermitteln sein. Bei Raja clavata wird sie sicher am proximalen Ende schon frïh für längere Zeit sistirt, etwa in einem Stadium, welches dem in Fig. 4 abgebildeten entspricht. Hier sind die bleibenden Lamellen des späteren electrischen Kästchens (vgl. Fig. 5) bereits scharf von dem sublamellären, später sich zur alveolären Schicht entwickelnden Theil der Faser abgegrenzt. Will man nun nicht annehmen, dass in diesen Stadien auf der distalen Seite des Organs die Lamellen in dem Masse verschwinden, als sie auf der proximalen nen ge- 
bildet werden (oder umgekehrt), eine Annabme, für die ich keine Thatsachen anzuführen wïsste, so beweisen die Bilder, dass auf den Stufen, welche Fig. 4 und 5 darstellen, neue Lamellen nicht mehr angesetzt werden.

Von den zuerst gebildeten, also vom proximalen Ende der Fasern am weitesten entfernten Lamellenanlagen scheinen allerdings bei R. clavata nicht alle sich zu bleiberden Lamellen auszubilden. Wenn wenigstens alle Querschichten in dem keulenförmig verdickten Ende junger Fasern, die den Eindruck werdender Lamellen machen, sich wirklich zu bleibenden Blättern entwickelten, dann milsste die Zahl der letzteren in Stadien wie Fig. 4 und 5 grösser sein als sie es thatsächlich ist. In Fig. 2 und 3 kann man wobl 25 bis 30 Schichten zählen, welche den Eindruck werdender Lamellen machen, während in Stadien wie Fig. 4 und 5 und auch späterhin noch lange Zeit nicht mehr als 10 bis 12 , bäufig weniger gefunden werden. Unzweifelhaft werden also bei R. clavata viele Lamellenanlagen, bevor Stadien wie Fig. 5 erreicht sind, wieder zurückgebildet, indem sie allmählich in die den distalen Theil der Lamellenschicht bedeckende kernhaltige, später zur mächtigen alveolären Schicht sich ausbildenden metasarkoplastischen Lage gleichsam eingeschmolzen werden.

Einen weiteren Grund für die Annahme, dass die bleibenden Lamellen bei ihrer Ontogenese nicht die ganze Entwickelungsreihe von dem der rubenden Muskelsubstanz entsprechenden Zustand der Querstreifung an durchlaufen, liefert das Verhalten der jungen bleibenden Lamellen an ihrer Peripherie. Es vergrössern sicb nämlich die jungen Lamellen in seitlicher Richtung noch sehr lange und höchst beträchtlich. Dies Flächenwachsthum erfolgt bei R. clavata, wie unten näher gezeigt werden wird, wesentlich nur durch Anlagerung never Lamellensubstanz an der Peripherie. Die peripherischsten Stellen jeder Lamelle sind also die jüngsten. Man müsste demnach, wenn immer die ganze Phylogenese wiederholt würde, von der Peripherie nach dem Centrum der Lamellen fortschreitend, die verschiedenen Entwicklungsstadien einander folgen sehen. An Profilbildern guter, senkrecht durch die Lamellen geführter Schnitte sieht man aber auf Stadien wie Fig. 4 und 5 und auch späterhin noch, bis an die äusserste Peripherie in der Hauptsache dasselbe Bild wie im Centrum. Nur die Dicke der beiden Schichten, namentlich der metabolen, nimmt nach aussen 
hin $a b^{1}$ ), oft bis anf weniger als ein Drittel, aucb pflegt die fibrilläre Structur der letrteren Schicht dabei deutlicher zu werden (Fig. 5). Die Ontogenese der electrischen Lamellen ist demnach bei Raja clavata jedenfalls eine ziemlich stark abgekürzte Recapitulation der Phylogenese.

In späterer Zeit findet bei unserer Art offenbar wieder eine reichliche Neubildung von lamellöser Substanz auf der proximalen Endfläche der streifigen Schicht statt. Diese Lamellen erreichen aber nur geringere Dicke und laufen auch nicht, wie die anfangs gebildeten (s. Fig. 5) unabgebrochen über die ganze Breite des Organs weg, sondern scheinen mehr bruchstiickweise gebildet zu werden und zu wachsen, wie auch die Anfügung neuer Lamellensubstanz an der Peripherie dann nicht mehr im genauen Niveananschluss an die schon bestehenden Lamellen, sondern in wechselnder Höhe stattfindet. Hierdurch entsteht dann statt der regelmässig durchlaufenden Querstreifung, welche noch in Stadien wie Fig. 5 und auch später anfangs noch vorhanden ist, die unregelmässige "mäandrische" Zeichnung, welche besonders in dem proximalen und dem peripherischen Theil der lamellösen Schicht, wie schon M. Schultze's 2) Abbildung (a. a. O. Fig. 3) zeigt, die feinste und verwickelst verlaufende Streifung aufweist. Auf späteren Stufen erhält man auch den Eindruck, als ob an der unteren, dem alveolären Theil angrenzenden Schicht ein ähnlicher Process statttände, oder $o b$ die hier befindlichen Lamellen theilweise und in unregelmässiger Art in die Masse des kernfihrenden metasarkoblastischen Balkennetzes eingeschmolzen würden. Auch von diesem Verhältniss gibt schon $\mathrm{Max} \mathrm{Schultze's} \mathrm{citirte} \mathrm{Ab-}$ bildung eine gute Vorstellung.

Für $R$ aja radiata gilt nach den mir vorliegenden Präparaten in Uebereinstimmung mit $\mathrm{E}$ wa r's Abbildungen ohne Zweifel dasselbe wie für R. clavata insofern, als am proximalen Ende lange Zeit hindurch neue Schichten angesetzt werden und diese Schichten gleich bei ihrem Entstehen im Allgemeinen um so

1) Dasselbe Verhältniss bildet Ewart für Raja batis ab (a. a. 0 . Pl. 67. Fig. 8).

2) Max Schultze, Zur Kenntniss des den electrischen Organen ver. wandten Schwanzorganes von Raja clavata. Archiv f. Anat. Physiol.u. s. w. von Joh. Müller, Jahrg. 1858. p. 193. Taf, IX. 
mehr den Charakter der bleibenden Lamellen besitzen, je später sie gebildet werden.

Auch bei Raja circularis finde ich in der Blätterschicht des entwickelten Organs in dieser Beziehung wesentlich dasselbe wie bei R. elavata. Ewart's sehr genaue Abbildung (a. a. 0 . Pl. 68 Fig. 5) liess dies bereits erwarten.

Unser in der Ueberschrift dieses Abschnitts formulirtes. Resultat dürfte somit allgemeine Giltigkeit besitzen.

3. Bei der Umwandlung der quergestreiften Substanz in die Blättersehicht des elektrischen Organs ändern sich sowohl die Dimensionen als auch die morphologischen und physikaliseh-chemischen Eigenschaften der metabolen und arimetabolen schichten.

Schon der blosse Anblick von Objecten wie die in Fig. 1-5 und 7 abgebildeten lehrt, dass bei der Entwicklung der Faser zum electrischen Kästchen von Raja clavata die metabolen wie die arimetabolen Schichten in Ausdebnung zunehmen und zwar, dass das Flächenwachsthum, senkrecht zur Faserachse, weitaus am bedeutendsten ist. Uebertrifft die Flächenausdehnung der Lamellen doch schon in dem in Fig. 5 abgebildeten Zustand die der Querscheiben der ursprïnglichen Muskelfaser um weit mehr als das Anderthalbhundertfache. Aber auch die Höhe der Schichten zeigt eine Zunahme auf das Doppelte und Dreifache und dariber. 'Die Dickenzuahme betrifft beide Schichten, jedoch nicht in gleichem Grade.

Auch die Aenderungen im morphologischen und physikalisehchemischen Verbalten beider Lagen sind so auffallig, dass sie selbst einem mit der feineren Histiologie der quergestreiften Muskelfasern durch eigene Anschauung nicht vertrauten Beohachter schon bei flichtiger Prïfung mit mittelstarken Vergrösserungen schwerlich entgehen können.

Im gewöhnlichen Licht, oder zwischen parallelen Nicols untersucht, macht das Bild der ruhenden oder mässig kontrahirten (Fig. 1) Muskelsubstanz allmählich einem Bilde Platz, wie es die stark contrabirte Muskelfaser im Stadium d er U m k ehrung z eigt (Fig. 3, 4, 5): schmale, sehr dunkle Querstreifen, getrennt durch breite, sehr helle Bänder, in deren Mitte ein etwas dunklerer verwaschener Querstreif hinzieht.

Im polarisirten Licht bei wirksamster Orientirung geprüft, fällt 
besonders die bedeutende Schwäehung des Doppelbrechungsvermögens auf. Wie Fig. 1 a zeigt, ist bei Raja clavata die Anisotropie der metabolen Sehicht schon fast völlig unmerklich geworden, ehe noch irgend eine andere, auf die Umwandlung bezigliche Aenderung zu entdecken ist. Dieser wichtige Punkt wird weiter unten näher besprochen.

Wie bei $R$ aja clavata verhalten sich die Dinge in der Hauptsache offenbar auch bei R. eircularis. Meine Beobachtungen sind in dieser Beziebung in Uebereinstimmung mit Ewart's Abbildungen: die Lamellen werden in allen Dimensionen grösser, die arimetabolen ausserdem stärker, die metabolen schwächer lichtbrechend und, wie ich hinzufügen kann, isotrop.

Bei Raja radiata fehlt dagegen, wie schon oben erwähnt, die Höhenzunahme der Schichten. Die metabole Lage zeigt an den mir vorliegenden Präparaten sogar eine relativ starke Höhenabnahme, auch ist ihr Lichtbrechungsvermögen in den erwachsenen oder nahezu erwachsenen Organen nicht so gering. Die der Lamellenschicht entsprechende plattenförmige Endanschwellung. macht deshalb hier bei nicht sehr starker Vergrösserung den Eindruck einer ziemlich stark lichtbrechenden, homogenen, einzelne Kerne bergenden Masse. Das Polarisationsmikroskop lehrt, dass die Doppelbrechung zwar gleichfalls sehr bedeutend abnimmt, aber doch sehr viel langsamer als bei R. clavata.

Rücksichtlich der unbestimmten Art, auf welche sich unsere Fig. 7 bezieht, kann ich nur sagen, dass das Doppelbrechungsvermögen ihrer metabolen Schichten viel langsamer schwindet als bei R. clavata, etwa so schnell wie bei R. radiata, und dass die weitest vorgeschrittenen Stufen, welehe in unseren Präparaten zu finden sind, (etwa Fig. 7, VI gleich), keine nennenswerthen Aenderungen in Höhe oder Ausseben der Schichten zeigen im Vergleich zur quergestreiften Substanz in den nicht angeschwollenen, distalen, faserförmigen Theilen der Organanlagen.

4. Das. Flächenwachsthum der metabolen und arimetabolen Schicht beruht, wenigstens bis zur Ausbildung der Kästchenform, wesentlich nur auf Vermehrung der ZahI der Fibrillen, nicht auf Verdickung der bereits bestebenden Fibrillen oder Verbreiterung der interfibrillären Ränme. 
Genaue Prüfung und Messung bei stärksten Vergrösserungen lehrt, dass die Zahl der im selben Raum vorhandenen Fibrillenelemente sich während des anfänglichen Wachsthums der Schichten nicht merklich ändert und dass auch die Dicke dieser Elemente wenigstens in der metabolen Schicht, und somit auch die Weite der mit "Sacroplasma" gefüllten interfibrillären Räume, zunächst merklich dieselbe bleibt. Man vergleiche Fig. 1a, b, 2a, 3a, $4 \mathrm{a}$. Auch auf späteren Entwickelungsstufen (Fig. 5), wo die Lamellen bereits eine hobe Ausbildung erfahren haben, ihr Flächenmass auf das Hundertfache und darüber gewachsen ist, kann man bei guter centraler oder schiefer Beleuchtung mit den besten starken Objectiven noch deutlich die Stäbchen in der metabolen Schicht erkennen und findet sie dann zwar länger, aber nicht merklich dicker oder weiter auseinanderstehend (Fig. 5a). Die etwa vorhandenen Unterschiede sind in keinem Falle so, dass sie für die Erklärung der zunehmenden seitlichen Ausdehnung der Lamellen in diesen Stadien irgendwie in Betracht kommen könnten.

Auf späteren Entwickelungsstufen wie auch im ausgewachsenen Organ vermochte ich an unseren Balsampräparaten von einer fibrillären Structur der Lamellen, auch der metabolen, keine sicheren Spuren mebr zu finden (Fig. 6), so dass ich unentschieden lassen muss, ob auch weiterhin das Flächenwachsthum nur auf Vermehrung der Fibrillenzahl beruht. Es ist wohl möglich, dass bei passenderer Erhärtung, etwa in Salicylsäure, dünnem Alkohol oder dünner Chromsäure und bei Untersuchung in Wasser oder schwachem Glycerin, auch in späteren Stadien noch Andeutungen einer Zusammensetzung aus Fibrillenelementen kenntlich sein werden. Auch Flächenbilder wären zu prifen.

Bei Raja circularis vermag ich in dem etwa $E$ wart's Taf. 68 Fig. 5 entsprechenden, weit entwickelten Stadium ausser einer zweifelhaften Körnelung der arimetabolen Lamellen keine Andeutungen fibrillärer Structur zu entdecken.

Dagegen gelingt dies leicht selbst an den weitest ausgebildeten Organen von R. radiata, die ich Ewart's Güte verdanke. Namentlich die dunklen arimetabolen Lamellen erscheinen an vielen Stellen deutlich aus gleichgrossen, zu einfacher Schicht nebeneinander gereihten Körnchen zusammengesetzt. Auch in den metabolen Lagen wird an mehreren Stellen durch die besten Objective eine Andeutung der fibrillären Structur enthüllt. Sicher ist, dass 
weder die Dicke der Fibrillenglieder noch ihre seitlichen Abstände grösser sind als im typisehen Muskelfaserzustand, eher kleiner, wie ja auch ibre Länge bedeutend abgenommen hat. Das Flächenwachsthum beruht also auch hier, und in noch höherem Grade als bei R. elavata, auf einer Vermehrung der $\mathrm{Zahl}$ der Fibrillenglieder, nicht etwa auf einer Quellung schon vorbandener Elemente.

5. Die Vermehrung der Zahl der Fibrillenelemente, auf welcher das Flächenwachsthum der Lamellen berubt, findet hauptsächlich, wo nicht ausschliesslich an der Peripherie der Lamellen statt.

Wenn man solche Organanlagen, in denen (wie z. B. in Fig. 7) die fibrilläre Structur sehr deutlich ist, sehr sorgfältig mit starken Vergrösserungen untersucht, so ergibt sich, wenigstens für R. clavata und die Art, auf welche sich Fig. 7 bezieht, dass die den faserförmigen Theil der Anlage zusammensetzenden quergestreiften Fibrillen sich geradlinig, parallel der Längsaxe der Faser in die keulenförmige Endanschwellung bis an deren proximales Ende fortsetzen, nicht etwa beim Eintritt in dieselbe auseinander weichen. Denselben geradlinigen, der Faseraxe parallelen Lauf besitzen auch alle Fibrillen in den peripherischen Theilen der Endanschwellung, bis an das Sarcolemm heran. Die oberflächlichsten Fibrillen sind also hier zugleich die kürzesten. Es lassen sich keine Beweise dafür finden, dass neue Fibrillen sich durch Längsspaltung älterer bilden: die Fibrillenglieder sind überall in der Lamelle merklich gleichgeformt und gleich dick.

Die schon von früheren Beobachtern hervorgehobene Abwesenheit von Kernen im Innern der mäandrischen Schicht von $R$. c lavata spricht auch dagegen, dass hier eine Neubildung von Fibrillen erfolge. An der Peripherie dagegen ist die lamellöse Schicht dauernd von einem kernhaltigen Protoplasmamantel bekleidet, welcher gewiss die Rolle einer Matrix spielen kann ${ }^{1}$ ).

$\mathrm{Ob}$ bei den Arten von Raja, welche wie nach Ewart R. radiata und eircularis auf die Dauer Kerne in der lamellösen Schicht besitzen, auch nur peripherisches Wachsthum erfolgt, oder ob hier nicht vielleicht, wie wahrscheinlicher, auch

1) S. auch die Abbildungen bei Ewart, a. a. 0. P1. 67. Fig. 13. Pl. 80. Fig. 12A und bei Muskens, a. a. 0. Pl. I. Fig. 46, 5c-e, 6. 
im Innern der Lamellen neue Fibrillen eingefügt werden, mag späteren Untersuchungen zur näherer Entscheidung überlassen bleiben. In den "clubs" von R. radiata ist der Verlauf der Fibrillen jedenfalls nicht so regelmässig parallel der Faseraxe wie bei R. clavata und laufen speciell auch viele Fibrillen und Fibrillensäulchen an der Peripherie beim Uebergang aus dem cylindrisehen in den kolbigen Theil der Faser nach aussen convex gebogen, der Oberfläche parallel weiter.

Raja batis, welche schon früh keine Kerne mehr in der Blätterschicht aufweist, verhält sich nach $\mathrm{E}$ warts Abbildungen (u. a. 0. Pl. 66. Fig. 6) zu urtheilen, wie R. cl a va ta. Bei diesen beiden Arten wird also, wenigstens so lange die fibrilläre Structur nachweisbar bleibt, nur von einem Flächenwachsthum der Lamellen durch Apposition, nicht durch Intussusception gesprochen werden können. Ein principieller Unterschied soll hiermit nicht bezeichnet sein, da anch im anderen Falle Apposition an vorhandene Fibrillen stattfindet, wie denn ja jedes Wachsthum durch sogenannte Intussusception wesentlich Apposition ist, wenn man nur mit der Betrachtung weit genug zu den kleinsten Theilchen hinabsteigt.

6. Die Dickenzunabme der Lamellen beruht a u f Verlängerung, die Dickenabnahme a uf Kürzerwerden der Fibrillenglieder beider Schichten, namentlich der metabolen.

Es mag zum Belege dieser Sätze auf die Abbildungen Fig. 1 a bis 5 a verwiesen werden. Neue, durch selbständige Querlinien begrenzte Lagen treten, wie diese Figuren zeigen, bei R. clavata bei fortschreitender Dickenzunahme der Lamellen nicht auf. Die vorhandenen Elemente verlängern sich nur, und zwar ist kein Zweifel, dass die Verlängerung bei $R$. elava $t$ a in stärkerem Grade die metabolen als die arimetabolen Glieder betrifft. Für R. batis und circularis gilt dasselbe. Bei R. radiata erscheinen dagegen, wie schon erwähnt, die Lamellen späterer Entwickelungsstufen wenigstens an den mir vorliegenden Präparaten viel niedriger, namentlich die metabolen, was in Verband mit den in den vorigen Abschnitten mitgetheilten Thatsachen nur auf einer Verkürzung der einzelnen Fibrillenglieder beruhen kann.

Bei der Beurtheilung dieser Dickenänderung ist der Einfluss im Auge zu behalten, welchen gleichzeitige A e nderunge n des pbysiologischen Contractionszustands auf die ab- 
solute and relative Länge der beiden Arten von Gliedern möglicherweise haben können. Da nach $\mathrm{Bab} \mathfrak{a} \mathrm{ch}$ i $\mathrm{n}^{1}$ ) bei gewissen nicht näher bestimmten Raja-Arten die Muskelfasern noch in den keulenförmigen Zuständen unserer Fig. 2, 3, 7 sich auf ,galvanische Reizung" verkürzen können, bei der Contraction der Muskelfaser aber die Höhe beider Schichten abnimmt und zwar bekanntlich die der arimetabolen viel schneller als die der metabolen, so begreift man, dass hierdurch zu einer erheblichen Complication der Erscheinungen Anlass gegeben werden kann. Es wird durch Versuche an lebenden Objecten festgestellt werden müssen, bis zu welcher Stufe der Ausbildung bei jeder Art die sich entwickelnden Lamellen noch merkliches Contractionsvermögen besitzen. Wir kommen auch auf diesen wichtigen Punkt noch zurïck.

7. Mit zunebmender Ausbildung der Lamellen wird die arimetabole Schicht optisch homogener, als Ganzes stärker lichtbrechend, dabei fester.

In den frühesten Zuständen, wie Fig. 7 a, erkennt man in der noch breiten isotropen oder arimetabolen Schicht die für die ruhende Muskelsubstanz der Vertebraten typische Zusammensetzung aus einer dünnen dunklen Mittellinie $(Z)$, der sog. Kraus eschen Linie, und zwei hellen, breiten, seitlichen Bändern $(J)$, welche $Z$ von den dunkleren, doppelbrechenden Querscheiben trennen. Wegen der sehr geringen Höhe der Fächer ist, wie ich bei einer früheren Gelegenheit ${ }^{2}$ ) bemerkt habe, hier nicht sicher, zu sagen, ob die dunkele Linie $Z$ bloss der Zwischenseheibe oder, was wahrscheinlicher, der Summe von Zwischenscheibe, zwei angrenzenden, plasmatischen Schichten (E nach Rollett) und zwei Nebenscheiben (N) entspricht.

Fig. 1 a, von R a ja clavata, steht bereits an der Grenze des Uebergangs- oder homogenen und des Umkehrungsstadiums: die arimetabole Schicht ist erheblich niedriger als die metabole, $J$ nicht mehr als selbständige Lage zu unterscheiden, $Z$ etwas verdickt, dabei noch dentlich aus einzelnen, den Fibrillengliedern entsprechenden Körnchen zusammengesetzt. Fig. $1 \mathrm{~b}$ und $2 \mathrm{c}$, noch mehr $3 \mathrm{a}$ und die folgenden zeigen das typische Bild des Umkeh-

1) Arch. f. Anat. Physiol. u. s. w. 1876. p. 535.

2) Mikrosk. Untersuch. üb. die quergestr. Muskelsubstanz. Arch. f. d. ges. Physiol. u. s. w. von P flüger. Bd. 7. 1873. p. 36 flg. 
rungsstadiums : eine einzige dunkle isotrope Schicht, deren Dicke viel zu gross ist, als dass sie der ganz unveränderten Zwischenscheibe entsprechen könnte. In Figur 3 a ist die Zusammensetzung aus Körnchen noch gut kenntlich, in Fig. 5 a und 6 nicht mehr. In den beiden letzten Figuren ist dagegen jedes dunkle Band aus einer mittleren, sehr dunklen glatten Linie und zwei sie begrenzenden, durch einen kaum merklichen Zwischenraum von ihr getrennten helleren, schwach körnigen Säumen zusammengesetzt. Man wird wohl annehmen dürfen, dass die mittlere dunkle Linie der stärker entwickelten $Z$ wischenscheibe, die beiden Säume hauptsächlich den ebenfalls gewachsenen Nebenscheiben (mit Einschluss von E) entsprechen.

Zwischen gekreuzten Nicols unter einem Winkel von $45^{\circ} \mathrm{zu}$ den Polarisationsebenen gelagert, schienen die dunklen Lamellen zuweilen schwach doppelbrechend zu sein, und mit einem Gypsplättchen in Roth erster Ordnung geprüft erwies sich die Doppelbrechung dann positiv in Bezug auf die Längsaxe der Fibrillenelemente. Es ist aber äusserst schwierig hier Gewissheit zu erlangen, ob die Doppelbrechung wirklich von der arimetabolen Schicht herrührt. Da die Lamellen in Stadien wie Fig. 5 and später wegen ibres wellig gebogenen Verlaufs fast nie genau senkrecht zur Ebene des Gesichtsfelds stehen, sind die aus ihnen in das Auge des Beobachters gelangenden Strahlen meist unter oder über der Einstellungsebene durch eine mehr oder minder dicke Schicht metaboler Substanz gegangen. Diese selbst aber ist, wenn auch nur in äusserst geringem Grade, sicher in gleichem Sinne doppelbrechend.

Die Präparate von Raja c i r c u la ris zeigen in Bezug anf die arimetabole Schicht wesentlich dasselbe, wie die von R. clavata. Nur liessen dieselben, wie schon unter 3 . erwähnt, auf noch weiter vorgeschrittenen Stufen in einem undeutlich körnigen Aussehen eine Andeutung der fibrillären Structur erkennen. Bei Raja radiat a erbält sich die letztere, wie oben gleichfalls schon bemerkt, sogar bis in den völlig entwickelten Zustand sehr ausgeprägt.

Die beschriebenen optischen Aenderungen der arimetabolen Schicht weisen darauf, dass sie mit steigender Ausbildung wasserärmer wird. Dazu stimmt auch die ziemlich grosse Festigkeit der entwickelten Lamellen, welehe sich bei Versuchen, dieselben bei 
R. clavata unter dem Mikroskop mit Nadeln zu zerreissen, bemerkbar machte.

Alles in allem genommen zeigt der Verlauf der Aenderungen, vom Verhalten der absoluten Dimensionen bei R. elavat $\mathrm{a}$, batis und circularis abgesehen, die grösste Aehnlichkeit mit denjenigen, welche bei starker physiologischer Verkürzung auftreten: di e arimetabolein Lamellen des fertig angelegten electrischen Kästchens zeigen viele Aehnlicbkeiten mit den sogenannten "Contractionsscheiben" des Umkehrungs. stadiums.

8. Mitzunehmender Ausbild ung derLamellen wird die metabole Sehicht, speciell die der Querscheiben, optisch homogener und schwächer lichtbrechend. Ibr Doppelbrechungsvermögen schwindet.

Der Verlauf dieser Veränderungen ist tür Raja clavata in den Figuren 1-5 dargestellt. Im Grossen und Ganzen ist auch der Verlauf dieser Aenderungen auffallend ähnlich dem bei der physiologischen Contraction. Und zwar gilt dies, trotz der oben schon angedeuteten Abweichungen auch fiur R. radiata, und wenn man von den entwickelten Zuständen schliessen darf, ebenso für R. circularis, wie nach Ewarts Abbildungen auch für R. batis. Für die Betrachtung in gewöhnlichem Licht ist die Aehnlichkeit so gross, dass Bilder wie unsere Fig. 3 und 4 eben so gut für quergestreifte Muskelsubstanz im Stadium der Umkehlung wie für Anlagen der mäandrischen Schicht der electrischen Kästchen gelten könnten. Speziell äussert sich die Verwandtschaft besonders deutlich bei $R$. clavata - noch weiter darin, dass das in der ruhenden Muskelsubstanz zwischen den beiden Querscheiben liegende hellere Band (Hen se n'sche oder Mittelscheibe, vgl. Fig. 7a) in den bleibenden Lamellen dunkler als die Querscheiben erscheint (Fig. 3-5).

Um so wichtiger ist es aber, die Unterschiede zu betonen. Oben wurde bereits hervorgeboben, dass die metabolen Fibrillenglieder nicht wie bei der Contraction kürzer und dicker, sondern im Gegentheil länger (ausser bei R. radiata und dabei nicht dicker werden. Dazu kommt nun als nicht minder wichtig der Schwund des Doppelbrechungsvermögens.

Zwar nimmt auch bei der physiologischen Verkürzung, wie 
V. von Ebner und nach ihm A. Rollett überzeugend nachgewiesen haben, die Kraft der Doppelbrechung der metabolen Schicht sebr merklich ab. Der Betrag dieser Schwächung ist jedoch nichtssagend im Vergleich zu dem bei der Umwandlung der Muskelfasern in die mäandrische Schicht der electrischen Kästchen. Bei Raja clavata verschwindet, wie aus Fig. 1 a bis 5 a ersichtlich, die polarisirende Wirkung fast vollständig und, was ganz besonders hervorgehoben werden muss, dieser Sehw und tritt schon ein, ehe überhaupt die Umwandlung der Faser in die Anlage eines electrischen Kästchens sich auf irgend eine andere $W$ eise anmeldet, also anch ehe das proximale Ende der quergestreiften Faser sich keulenförmig za verdicken beginnt. Schon in so jungen Zuständen wie Fig. 1 war nur noch eine sehr schwache Wirkung zwischen den gekreuzten Nicols oder im farbigen polarisirten Lichte zu bemerken. Dass die Doppelbrechung nicht durch die Präparationsweise geschwächt war, darf bestimmt behauptet werden, denn die in denselben Präparaten vorhandenen echten Muskelfasern waren sämmtlich stark doppelbrechend geblieben, sämmtliche zur Umbildung in electrische Kästchen sich anschickende Fasern, auch die jüngsten Stadien dagegen relativ unwirksam.

Fig. 2a, 3a, 4a, welche Stellen der keulenförmig angeschwollenen Faserenden wiedergeben, zeigen, wie auch Fig. 5 a, trotz der bedeutenden, auf Vermebrung der Fibrillenzahl beruhenden Dickenzunahme, doch bei gleicher wirksamster Orjentirung, keine steigende, sondern eine abnehmende Helligkeit auf dunklem Grunde verglichen mit der viel dünneren Faser Fig. 1. Ja, es bedurfte bei diesen ziemlich dicken Objecten schon sehr guten Lichts, um überhaupt eine Wirkung zu bemerken. Bei Anwendung schwacher Vergrösserungen $(50-100 \mathrm{mal})$ genügte helles diffuses Tageslicht. Für die Untersuchung mit starken Immersionssystemen war dies bei weitem nicht hinreichend. Electrisches Gliblicht, in friiher ${ }^{1}$ ) von mir beschriebener Weise erzengt und mittels des Abbe'schen Condensor nach vorheriger Polarisation auf das Object concentrirt, ergab die besten Resultate. An Längsschnitten von nur etwa

1) Th. W. Engelmann, Die Farben bunter Laubblätter und ihre Bedeutung für die Zerlegung der Kohlensäure im Lichṫe. Onderzoek. physiol. lab. Utrecht. 3. R. D. X, 1887. p. 155 flg. - Botan. Zeitg. 1887. No. 25-29. 
0,015 mm Dicke oder darunter konnte ich bei keiner Beleuchtung deutlicbe Wirkung entdecken.

Bei Kästchenanlagen von der in Fig. 5 abgebildeten und dieser folgenden Entwickelungsstufen war die Doppelbrechung an der Peripherie der Lamellen, wo diese geringere Dicke haben und nach unseren früheren Ausführungen jünger sind, entschieden stärker als in der Mitte. Hierin darf man gleichfalls eine Bestätigung ihres geringeren Alters erblicken.

Bei Raja circularis haben an den mir von Ewart iibersandten Präparaten im ziemlich entwickelten Organ ${ }^{1}$ ) die metabolen Lamellen im Wesentlichen dasselbe Aussehen wie bei R. elavata. Von Doppelbrechung kann ich aber nicht die geringste Spur mehr entdecken. Die Schnitte sind freilich sehr dünn, wohl sämmtlich unter $0,01 \mathrm{~mm}$. Doch leuchten Muskelfasern und Bindegewebsbiindel im selben Präparat sehr stark zwischen gekreuzten Nicols.

Höchst bemerkenswerth ist das sehr viel langsamere und weniger vollständige Schwinden des Doppelbrechungsvermögens bei R. $r$ adiata. Hierin, wie in so manchen anderen schon bemerkten Punkten verräth sich, dass die Organe bei dieser Art einen phylugenetisch jüngeren Zustand darstellen. Die frühesten Stufen in E w a $\mathrm{t} s$ Präparaten, welche ziemlich genau den Fasern II und III unserer Fig. 7 entsprechen, zeigen wie diese das Doppelbrechungsvermögen im Vergleich zu dem der benachbarten embryonalen Muskelfasern zwar entschieden geschwächt, aber doch sehr viel weniger als in denselben Stadien bei $R$. cl a vata. In den Ewarts Taf. 79 Fig. 5-7, Taf. 80 Fig. 8 entsprechenden Phasen leuchten die ,clubs', wie in den in Fig. 7 abgebildeten Objecten unbekannten Ursprungs, unter $45^{\circ}$ gegen die Polarisationsebene des gekreuzten Nicols orientirt, hell auf, heller als die faserförmigen, freilich auch viel dünneren distalen Theile der jungen Organanlagen, obschon nicht so hell wie etwa halb so dicke benachbarte Muskelfasern. In Roth erster Ordnung mit Gypsplättchen geprïpft erscheinen sie gelbroth, bezw. purpurviolett, an den dicksten Stellen rein gelb, bezüglich rein blan. Die Wirkung setzt sich bis in die Stiele der Organe, ja bis ans distale Ende der Fasern fort. Benach- 
barte dünnere Muskelfasern leuchten unter gleichen Verhältnissen rein und hellgelb, bez. rein und hellblau, also viel stärker.

Auch die lamellöse Schicht der älteren Organe, welche einem Rochen von $7 \frac{1}{2} \mathrm{~m}$ Schwanzlänge entstammen, ist noch sehr merklich und in gleichem Sinne wie zuvor doppelbrechend.

In dem noch älteren, von einer R. radiata von 10 inch. Schwanzlänge herriuhrenden Präparate ist nur noch eine Spur von Anisotropie zu entdecken. Trotz grösserer Dicke der wirksamen Schicht ist bestenfalls nur ein matter Lichtschimmer im dunklen, oder ein schwacher Stich ins Gelbrothe beziuglich ins Violette im rothgefärbten Gesichtsfeld zu bemerken.

\section{Theoretische Bemerkungen.}

Wir baben nun am Ende unserer Untersuchung zu prüfen, ob die gefundenen histiologischen Thatsachen sich für die Physiologie der Muskeln und der electrischen Organe verwerthen lassen. Zunächst erhebt sich die Frage, ob zwischen dem Structurwechsel, auf welchem, wie wir sahen, die Umbildung der quergestreiften Substanz in die Blätterschicht des electrischen Organs beruht, einerseits und dem Functionswechsel andererseits im Einzelnen causale Beziehungen sich nachweisen lassen, die etwa näheres Licht auf Sitz, Ursprung und Wesen der Contractilität nnd der Electricitätsentwickelung werfen.

Die Frage sei in erster Linie mit Rücksicht auf die Contractilitätserscheinungen geprüft.

Der in dieser Beziehung wichtigste Fund besteht, wie ich glaube, in dem Verhalten des Doppelbrechungsvermögens der metabolen Schicht, insofern darin eine nene Stütze für die Annahme gelegen ist, dass nur die doppelbrechenden metabolen Glieder der Muskelfibrillen Sitz und Quelle der verkürzenden Kräfte des Muskels sind. Und zwar ist es nicht bloss die allgemeine Thatsache, d as s das Doppelbrechungsvermögen wäbrend der Ausbildung der electrischen Organe abnimmt, welche diese Annabme stïtzt, sondern es sind gerade die besonderen, dies Schwinden betreffenden Thatsachen, namentlich die Unterschiede, welche darin bei verschiedenen Arten von Raja bestehen, wie ich glaube, von grossem theoretischen Werthe. 
Während bei den einen Arten (R. clavata, wohl auch R. bat is) das Doppelbrechungsvermögen schon äusserst frïh fast unmerklich wird, ehe noch sonst ein Zeichen der bevorstehenden Umwandlung in ein electrisches Organ an der Muskelfaser bemerkbar ist, erhält es sich bei anderen Arten ( $R$ aja radiata, die unbestimmten Arten welchen $\mathrm{Bab}$ u $\mathrm{ch}$ in s Fig. 11 und 12 und unsere Fig. 7 entstammen) viel länger. Bei den letzteren Arten erhält sich auch in anderer Beziebung die ursprüngliche Muskelstruetur viel länger: unsere Fig. 7 zeigt noch auf ziemlich vorgeschrittener Stufe der Entwickelung das Bild der normalen ruhenden Muskelsubstanz. Bei R. r a d i t a macht selbst in fertigen Organ die Blätterschicht durchaus den Eindruck quergestreifter Muskelsubstanz, freilich sehr stark contrahirter. Bei jenen anderen Arten wird schon sehr frïh das Bild des Umkehrungsstadiums sichtbar und ist im völlig ausgewachsenen Zustand die Aehnlichkeit mit einer Muskelfaser so vollständig vertilgt, dass bei keinem der zahlreichen vortrefflichen Forscher, die vor $B$ abuchin das Schwanzorgan der Raja untersuchten, der Gedanke an genetische Beziehnngen zwischen beiden Gebilden ernstlich anfgenommen zu sein scheint.

Schon jetzt ist es wahrscheinlich, dass den Unterschieden im Schwinden der Doppelbrechung, welche die verschiedenen Species von Raja zeigen, Unterschiede im Contractionsvermögen parallel gehen, derart, dass in dem Maasse als die Doppelbrechung früher und vollständiger schwindet, auch die Contractilität früher und vollständiger verloren geht. $\mathrm{B} \mathrm{a} \mathrm{b} \mathrm{u} \mathrm{c} \mathrm{h} \mathrm{in} \mathrm{giebt} \mathrm{ausdrücklich} \mathrm{an,}$ dass er bereits stark keulenförmig angeschwollene Fasern auf galvanische Reizung sich noch contrahiren sah, und dies waren doch wohl Fasern derselben Raja-Art, auf welche auch Babuchins Figuren 19 und 20 sich beziehen, in welchen die bereits weit ausgebildete Lamellenschicht noch starke Doppelbrechung zeigt.

Ew a r t vermuthet bei $R$. r adiata eine lange Fortdaner des Contractionsvermögens. Ich möchte ausser dem langsameren Sinken des Doppelbrechungsvermögens für die Richtigkeit dieser Vermuthung noch den Umstand anführen, dass das Bild der quergestreiften Substanz bei R. radiata, im Gegensatz zu den Befunden bei R. elavata, selbst auf ganz gleichen Entwickelungsstufen, ja selbst an der nämlicben Faser, häufig ähnliche Unterschiede in Bezug auf Höhe und Aussehen der Schichten aufweist, wie sie 
bei echten Muskelfasern, auch in denselben Präparaten gewöhnlich sind und wie sie wesentlich nur dureh verscbiedene pbysiologische Contractionszustände bedingt sein können.

Leider feblt es durchaus an experimentellen brauchbaren Angaben. Es wird nöthig sein, systematische, vergleichende Reizversuche an möglichst vielen, durch ihr Verhalten in Bezug auf Doppelbrechung verschiedenen Objecten bei verschiedenen Raja-Arten anzustellen. Vor Allem wird es interessant sein za ermitteln, einerseits $o b$ quergestreifte Fasern, etwa der Art, wie in Fig. 1, von R. clavata, welche ihre Anisotropie schon nahezu völlig verloren, übrigens aber ganz das Aussehen functionsfähiger quergestreifter Muskelfasern behalten haben, noch zucken können, oder - wie ich vermuthe bereits zuckungsunfähig sind; andererseits ob da, wo die ausgebildeten metabolen Lamellen noch sehr merklich und regelmässig anisotrop sind (z. B. bei halb erwachsenen R. radiata), noch Contractilität experimentell nachweisbar ist, wie ich für wahrscheinlich halten muss und anch $E$ wart ${ }^{1}$ ) vermuthet. Der Experimentalforschung öffnet sich hier ein weites und gewiss lobnendes Feld.

Noch in einer anderen Beziehung scheinen mir die von uns gefundenen, das Schwinden der Doppelbrechung betreffenden Thatsachen theoretisch von Gewicht. Ich meine speciell den Umstand, dass zwar die Anisotropie aus den metabolen Fibrillengliedern verschwindet, diese selbst aber zunächst übrigens ganz unverändert bestehen bleiben, ja anfangs nicht einmal eine merkliche Aenderang ihres Brechungscoefficienten erleiden. Diese Thatsache ist in schöner Uebereinstimmung mit der frither von mir entwickelten Vorstellung, dass die Muskelfibrillen im Wesentlichen aus einer isotropen, in der Längsriehtung durchlaufenden Grandsubstanz")

1) a. a. o. p. 549 .

2) Ich habe diese Grundsubstanz der Fibrillen keineswegs, wie Rollett meint, für identisch mit dem interfibrillären isotropen Sarcoplasma, sondern immer für eine den Fibrillen eigenthümliche, organisirte Substanz gehalten. (Vgl. dies Archiv. Bd. XI. 1874. pag. 462. - Bd. XXV. 1881. pag. 542 f.) Ueberhaupt wüsste ich kaum, in welchem wesentlichen Punkte eine ernste Differenz zwischen der Auffassung meines verehrten Collegen and derjenigen bestände, die ich seit nummehr bald einem Vierteljahrhundert vertheidigt habe. 
bestehen, in welche in regelmässigen, den metabolen Gliedern entsprechenden Schichten, doppelbrechende, als Sitz der verkürzenden Kräfte zu betrachtende Theilchen eingelagert sind. Sie ist ebenso in Uebereinstimmung mit der neuerdings ${ }^{1}$ ) ron mir dargelegten Vermuthung, dass die contractilen doppelbrechenden Theilchen nur einen sehr kleinen Bruchtheil der Masse der sarcons elements bilden. Ich darf hierin eine neue Stütze für meine Theorie vom thermodynamischen Ursprung der Muskelkraft erblicken, welche verlangt, dass nur ein sehr kleiner Bruchtheil der gesammten Muskelmasse die zur Erzeugung: der mechanischen Arbeit erforderliche ansehnliche Temperatursteigerung. erfäbrt. Beständen die metabolen Fibrillenglieder (sarcous elements) - vom eingelagerten Wasser abgesehen - durchweg aus doppelbrechenden Micellen, so würde das Verschwinden des Doppelbrechungsvermögens in unserem Falle gewiss nicht obne sonstige merkliche Aendẹrungen in Dimensionen, Form oder Brechungsvermögen stattfinden können, eher wohl ein gleichzeitiges völliges Zugrundegehen der metabolen Glieder der Fibrillen zu erwarten sein. Dies erfolgt aber, wenn überhaupt, erst in viel späteren Entwickelungsperioden.

Nicht bloss das Schwinden der Doppelbrecbung, auch die im gewöhnlichen Licht bemerklichen, an das Auftreten des Umkehrungsstadiums bei der Contraction erinnernden Aenderungen der optischen und mechanischen Eigensebaften der metabolen und arimetabolen Schichten dürfen wohl mit dem allmählichen Schwinden der Contractilität in Verband gebracht werden. Denn, gleichviel welche Vorstellung man sich über das Wesen des Contractionsvorgangs machen möge, sicher ist seit S chwan n S Versuchen, dass in dem Maasse als die Verkürzung weiter vorgeschritten ist; die Fähigkeit zur Entwickelung neuer verkürzender Kräfte abnimmt. Nach meinen frïheren Messungen ${ }^{2}$ ) tritt aber das Bild des Umkehrungsstadiums immer erst bei sehr hohen Verkürzungsgraden auf, bei

1) Ueber den Ursprung der Muskelkraft. Leipzig 1893. Zweite Aufl. p. $28 \mathrm{fg}$. - Ueber einige gegen meine Ansicht vom Ursprung der Muskelkraft erhobene Bedenken. Arch. f. d. ges. Physiol. u. s. w. von Pflüger, Bd. 54. 1893. p. 637.

2) Neue Untersuchungen über die mikroskopischen Vorgänge bei der Muskelcontraction. Arch. f. d. ges. Physiol. von Pflüger. Bd. 18. 1878. p. 13. - Mikrometrische Untersuch, an contrahirten Muskelfasern. Ebda. Bd. 23. 1880. p. 571. 
Käfermuskeln etwa wenn die Länge des ruhenden, mässig gedehnten Faserelements auf die Hälfte gesunken ist. Da nach meiner Theorie der Contraction bei der Verkürzung Wasser aus der arimetabolen in die metabole Schicht treten muss, begreift es sich, dass die mit der Ausbildung des Umkehrungsstadiums zunehmende, in dem Auftreten der dunklen "Contractionsscheiben" sich äussernde Wasserverarmung der arimetabolen Schicht zu einer steigenden Schwächung des Contractionsvermögens Anlass geben muss. Der Wasservorrath wird erschöpft.

Auch auf diese Punkte wird. bei Reizversuchen Rücksicht zu nehmen und im. Einzelnen zu prüfen sein, in wie weit das Verhalten der Contractilität beim Structurwechsel mit den hier angedeuteten theoretischen Vorstellungen in Uebereinstimmung ist. Solange es aber an ausdrücklich hierauf gerichteten experimentellen Untersuchungen fehlt, möchte es nicbt gerathen sein, diese theoretischen Betrachtungen weiter auszuführen.

Der Mangel experimenteller Daten macht sich in noch höherem Masse geltend bei der Prüfung der Frage, ob die von uns gefundenen histiogenetischen Thatsachen für die $T$ h e orie $d$ er electrischen Vorgänge in Muskel und electrischem Organ Verwerthung finden können. Ich möchte hier kaum bestimmte Vermuthungen wagen, sondern nur ganz im Allgemeinen darauf aufmerksam machen, dass das Bestehenbleiben und Wachsen der is o tropen Fibrillensubstanz, speciell auch die absolut (R. clav at a, batis c i i c u la ris) oder doch relativ (R. radiata) starke Ausbildung der den Zwischen- und Nebenscheiben entsprechenden Schicht bei der Umbildung der Muskelfaser zum electrischen Kästchen in guter Uebereinstimmung ist mit der früher von mir begriundeten Vorstellung, dass die electromotorischen Processe im Muskel an die reizleitende isotrope Substanz gebunden sind, und dass der Nerv zunächst auf die arimetabole Schicht einwirkt. In den electrischen Kästchen des Schwanzorgans von $R$ aja bat is, cl a vata u. a. liegen die isotrop gewordenen Lamellen unmittelbar unter der sogenannten Nervenendplatte, wie nach A. Fötting er's von mir bestätigten Beobachtungen bei Käfermuskeln die Zwischenscheiben zunächst unter den Enden der den Nervenhügel durchsetzenden Nervenfibrillen ${ }^{1}$ ).

1) Ich muss, gegenüber den negativen Befunden Rolletts, den von 
Zu prüfen wäre auch, ob nicht da, wo die zur Längsaxe senkrechte Querstreifung bis an die der proximalen Endfläche anfliegende protoplasmatische Schicht, die sogen. Endplatte hinanreicht, dasselbe Verhalten besteht, welches ich für Insektenmuskeln nachwies, dass nämlich die quergestreifte Substanz am Faserende mit einer halben arimetabolen Schicht endigt, die hier also in ihrer vollen Flächenausdehnung $z \mathfrak{u}$ den Nervenendästen in nähere Beziehung treten würde. Bilder, wie unsere Fig. 4 und 5 , sprechen entschieden dafür. Doch ist die Untersuchung schwierig. Wer weiss, ob nicht auch die von $R u d$. W a g n e r entdeckten Körner und die von $R$ em a k zuerst bemerkten palissadenartigen Stäbchen (cils électriques von Ranvier), welche in den electrischen Organen des Zitterrochens unmittelbar an die letzten Ausläufer der Nervenausbreitung sich anschliessen, den die Zwischen- und Nebenscheiben der Muskelfasern bildenden Fibrillengliedern gleichwerthig sind. Sicher ist, dass auch bei den electrischen Organen von Torpedo, M a lopterurus und Gymnotus die zunächst unter der terminalen Nervenausbreitung gelegene Schicht, wie überhaupt die Masse der sogenannten electrischen Platte keine oder nur vergleichsweise unmerkliche Spuren von Doppelbrechung zeigt ${ }^{1}$ ).

Andererseits ist jedoch auch die Frage berechtigt, ob die im Vergleich zu den electrischen Organen der eigentlichen Zitterfische viel geringere Grösse der electromotorischen Wirkung der Schwanzorgane von Raja nicht gerade mit der starken Entwickelung der lamellösen Schicht zusammenhängt ${ }^{2}$ ), welche ja eins der wesentlichsten histiologischen Unterscheidungsmerkmale beider Klassen von

Föttinger beschriebenen Sachverhalt aufrecht erhalten. Die Untersuchung ist schwierig und zeitraubend. Unter Hunderten von Nervenhügeln in Föttinger's Präparaten war kaum ein halbes Dutzend, welche mir beweisend schienen. Es ist zu hoffen, dass dieser wichtige Punkt an der Hand neuerer besserer Methoden, etwa nach Golgi oder Ehrlich, gründlich weiter untersucht wird.

1) Ueber den Bau der quergestreiften Substanz an den Enden der Muskelfasern. Arch. f. d. ges. Physiol. u. s. w. von Pfläger. Ba. 26. 1882. p. 531.

2) A. Babuchin, Uebersicht der neuen Untersuchungen u. s. w. Arch. f. Anat., Physiol. u. s. w. 1876. p. 541. E. du Bois-Reymond, Dr. Carl Sachs Unters. am Zitteraal, 1881. p. $61 \mathrm{flg}$. - G. Fritsch, die electrischen Fische, 2. Abth. Die Torpedinëen. 1890. p. 114. 
Organen bildet. Sollten die Lamellen dennoch an der Electricitätsentwickelung direct theilnehmen, so wäre vielleicht der unregelmässige, mäandrische Verlauf für die Schwäche der Wirkung nach aussen mit verantwortlich zu machen. Man wird doch eine auf morphologiseher Structur beruhende räumliche Orientirung der electromotorischen Kräfte schwerlich entbehren können und diese Orientirung in jedem Element in Bezug auf die Oberfläche der Lamellen naturgemäss als dieselbe vorauszusetzen haben. Wegen der regellosen Krümmungen der Lamellen müssten dann die Wirkungen der einzelnen Elemente, wie stark auch jede für sich sein möge, sich nach aussen hin sehr bedeutend schwächen. Es wäre zu prüfen, ob bei denjenigen Arten und namentlich auch auf denjenigen Entwickelungsstufen, wo die Lamellen noch wesentlich parallel und plan verlaufen (etwa wie bei Raja batis nach Ewart ${ }^{1}$ ), oder wie in unserer Fig. 5 von R. clavata), stärkere electrische Wirkungen nach aussen erhalten werden können immer unter Beriicksichtigung des Einflusses sonstiger Unterschiede in Bau, Dimensionen, Anordnung u. s. w. - als bei solchen Organen, die wie bei älteren R. elavata und R. circularis ${ }^{2}$, unregelmässig mäandrisch verlaufende Lamellen besitzen. Für erwachsene Raja batis besitzen wir bereits eine gründliche, nach den besten Methoden durchgefübrte Untersuchung von Burdon-Sanderson und Gotch ${ }^{3}$ ). Danach kann die Kraft des Schlags beim Organ des erwachsenen Thiers sebr beträchtliche Werthe ( $1 / 2$ Volt) erreichen. Hier laufen wirklich auch noch im entwickelten Organ nach Ewarts Abbildungen ${ }^{4}$ ) die Lamellen wesentlich parallel der Endfläche, keineswegs deutlich mäandrisch.

Mag man nun die Lamellen an der Electricitätsentwickelung direct betheiligt halten oder nicht, kein Zweifel kann darüber bestehen, dass die electrischen Leistungen der Organe von Raja auf dem jetzigen Zustand ihrer Ausbildung so geringe sind, dass diese Organe als electrische Vertheidigungsapparate oder Angriffswaffen nicht in Betracht kommen können. Insofern erscheinen sie also

1) a. a. 0. P1. 66 und 67.

2) Ewart, a. a. 0. Pl. 68. Fig. 5.

3) Burdon-Sanderson und F. Gotch, On the electrical organ of the skate. Journ. of Physiol. Vol. IX. 1888. p. 137-166.

4) a. a. 0. Pl. 67. Fig. 10 u. 13. 
ohne Nutzen für Individuum und Art zu sein. Es ist bekannt, welche principielle Schwierigkeit für Darwins Theorie der natürlichen Zuchtwahl in der allmählichen Entstehung und Ausbildung zunächst anscheinend durchaus nutzloser Organe erbliekt wird. Für Darw in selbst bildeten gerade die electrisehen und pseadoelectrischen $0 \mathrm{r}$ gane zeitlebens einen dunklen Punkt, auf den er in seiner strengen Gewissenhaftigkeit immer wieder zuriickkam. Auch Babuchins Entdeckungen des musculären Ursprungs unserer Apparate haben, wie schon E. $d u$ B o i s-Reymo n $d^{x}$ ) bemerkt, diese Schwierigkeit nicht beseitigt, wenn ihnen auch das andere Verdienst znkommt, uiber die Descendenz der Organe, ïber den Weg, den die Phylogenese morphologisch genommen, Aufschluss ertheilt zu haben.

„Die Schwierigkeit", sagt d u B o is-Reymond, „welche die vollkommenen electrischen Organe der Selectionstheorie bieten, wird durch die unvollkommenen nicht verringert. Die Wirkungen der letzteren sind der Art, dass sie dem Thier als Schutz- und Angriffswaffe von keinem Nutzen sein können; sie können sich also auch nicht durch Zuchtwahl zu vollkommenen electrischen Organen hinaufarbeiten. Wären sie aber im Stande, dem Thiere Nutzen zu bringen, so fände für sie dieselbe Schwierigkeit statt, wie für die vollkommenen electrischen Organe. In der phylogenetischen Reihe rückwärts gehend träfe man schliesslich auf so wenig entwickelte Stufen, dass die Organe noch von keinem Nutzen wären, also auch nicht durch Zuchtwahl sich vervollkommnen könnten $\left.{ }^{2}\right) . "$

Ich glaube, dass die Schwierigkeit heutzutage nicht mehr so unüberwindlich erscheinen darf, wo wir durch eine Reihe überraschender Erfahrungen an anderen Organen auf das Nachdrücklichste belehrt sind, wie bedenklich, ja wie anmassend es ist, die Nutzlosigkeit anscheinend rudimentärer, oberflächlich betrachtet sogar eher für schädlich als für nützlich zu haltender Organe bebaupten $z$ wollen. Ich habe selbstverständlich die theoretisch wie praktisch gleich folgenreichen Entdeckungen über die Bedeutung der Glandula thyreoidea und ähnlicher Organe, uiberhaupt

1) E. du Bois-Reymond, Dr. Carl Sachs Untersuch. u.s.w. p. $66 \mathrm{flg}$.

2) E. du Bois-Reymond, Dr. Carl Sachs Untersuchungen u. s. w. p. 68. - Vgl. auch desselben Autors Rede: Darwin versus Gallani. Monatsber. der Akad. v. Juli 1876. p. 396.

E. Pflüger, Archiv f. Physiologie. Bd 57, 
alle die überaus wichtigen Thatsachen im Auge, welehe zu der von Brown-Séquard neuerdings mit dem Namen der sécrétion interne bezeichneten Erscheinung in Beziehung stehen. Es handelt sich hier gewiss nicht um vereinzelte, als Ausnahmen zu betrachtende Fälle, sondern um ein allgemeines, für die Erhaltung des Individuums und der Art fundamental wichtiges Princip der correlativen Regulirung der Lebenserscheinungen, durch dessen Berücksichtigung und näheres Studium vielleicht jene grosse principielle Schwierigkeit für die Selectionstheorie ganz allgemein aufgehoben werden wird. Hat es angesichts dieser neven Errungenschaften etwas Unerlaubtes, anzunehmen, dass die Schwanzorgane der Rochen auf dem Wege der inneren Secretion für die Thiere von Vortheil, durch ihren ehemisehen Einfluss auf die Thätigkeit anderer Organe, - ich denke hauptsächlich an das Nervensystem - vielleicht geradezu unentbehrlich sind? Ja ist es nicht denkbar, dass der jenem Einfluss zu Grunde liegende Vorgang durch eine Art Cirkelprocess eine fortwährende Steigerung erfahren und damit auch die einmal angefangene, mit demselben causal verbundene morphologische Differenzirung eine fortschreitende Steigerung erfahren könne, derart, dass unsere Organe sich schliesslich doch vielleicht durch Zuchtwahl zu vollkommenen electrischen Organen hinaufarbeiten werden?

Ich möchte diese Bemerkungen und Fragen um so weniger unterdrücken, als sie der experimentellen Prüfung zugänglich sind. Man wird bei verschiedenen Arten von Raja, auf verschiedenen Stufen ontogenetischer und phylogenetischer Ausbildung zunächst den physiologischen Erfolg totaler und partieller Exstirpationen und Zerstörungen, mit und ohne nachfolgende Transplantation normalen electrischen Gewebes zu untersuchen haben. Vielleicht wird sich dabei sehr bald zeigen, dass und wozu diese angeblich nutzlosen Theile dem Thiere dienen, oder doch welche Nachtheile ibre Entfernung dem Gesammtorganismus bringt. Es eröffnet sich hier ein weites Feld für Versuche, zu dessen Bearbeitung die zoologischen Stationen unserer Meereskïsten gewiss günstige Gelegenheit bieten werden. 


\section{Erklärung der Abbildungen auf Tafel II.}

Fig. 1-6. Entwickelung des elektrischen Organs von Raja clavata. - Sämmt. liche Präparate stammen von einem in Pikrinschwefelsäure erhärteten Embryo von $7 \frac{1}{2} \mathrm{~cm}$ Länge.

Fig. $1(500 / 1)$. Sehr frühes Stadium. Beginn der keulenförmigen Verdickung des proximalen Theils der Muskelfaser.

Fig. La $(1500 / 1)$. Stück der Querstreifung aus der Mitte der Faser Fig. 1, die linke Hälfte im gewöhnlichen Licht, die rechte zwischen gekreuzten Nicols bei wirksamster Orientirung. Uebergangsstadium. Das Doppelbrechungsvermögen der metabolen Schicht bereits sehr stark vermindert (vgl. Fig. 1c).

Fig. $1 \mathrm{~b}\left({ }^{1500} / 1\right)$. Stück der Querstreifung aus der Mitte der Endanschwellung von Faser Fig. 1. Wie 1a. Die arimetabole Schicht etwas dunkler, die metabole in gewöhnlichem Licht heller als in Fig. 1a: Umkehrungsstadium.

Fig. 1c $(1500 / 1)$. Stück der Querstreifung eines Schwanzmuskelfasers desselben Präparats, von gleicher Dicke wie die Mitte der Faser Fig 1. Das Doppelbrechungsvermögen des metabolen Fibrillengliedes ist sehr viel stärker als in Fig. 1a und $1 \mathrm{~b}$.

Fig. $2(500 / 1)$. Späteres Stadium. Proximales Faserende stärker verdickt.

Fig. 2a $(1500 / 1)$. Stück aus der Endanschwellung von Fig. 2: Umkehrungsstadium weiter ausgebildet. Doppelbrechung noch merklich schwächer als in 1a. Dicke und Abstände des Fibrillengliedes unverändert. Länge grösser.

Fig. $3(500 / 1)$. Noch etwas späteres Stadium.

Fig. 3a $(1500 / 1)$. Stück der Querstreifung aus der Mitte der Anschwellung von Fig. 3. Die arimetabole Schicht dunkler und etwas homogener, die metabole im Ganzen heller geworden, nur in der Mitte etwas dunkler, mit noch deutlicher, obschon blasserer fibrillärer Structur.

Fig. $4(500 / 1)$. Uebergang zur Kästchenform. Die Blätterschicht am distalen Ende bereits ziemlich scharf von der nur noch undeutlich quergestreiften Substanz des faserförmigen Stiels der Organanlage abgegrenzt.

Fig. 4a $(1500 / 1)$. Stück aus der Mitte der Blätterschicht von Fig. 4. Wesentlich dasselbe Bild wie $3 \mathrm{a}$, doch ist die arimetabole Schicht noch dunkler und homogener geworden.

Fig. $5(400 / 1)$. Die Kästchenform ist erreicht, die Blätterschicht auf der proximalen und distalen Fläche scharf abgegrenzt. Lamellen stärker wellig gebogen, nach der Peripherie zu dünner und deutlich fibrillär. Noch keine Mäanderbildung. 
180 Th. W. Engetmann: Die Blättrschicht der electr. Organe etc.

Fig. 5a $\left({ }^{1500} / 1\right)$.' Stück aus der Mitte der Blätterschicht von Fig. 5. Arimetabole Schicht nicht mehr körnig, dagegen deutlich aus einer mittleren sehr stark lichtbrechenden und zwei seitlichen schwächer brechenden Lagen gebildet. In der sehr hell gewordenen metabolen Schicht die Fibrillen zwar sehr blass (ausgenommen die mittlere Partie) aber noch vollkommen deutlich. Dicke und seitlicher Abstand der Fibrillenglieder noch wie in Fig. 1a bis 4 a.

Fig. $6(150 \%)$. Optischer Querschnitt durch drei arimetabole Lamellen eines entwickelten Kästchens, im gewöhnlichen Licht: zeigt eine Zusam. mensetzung aus 3 Lagen.

Fig. $7(500 / 1)$. Längsschnitt durch ein Stück der electrischen Organanlage im Schwanz eines Embryos unbestimmter Herkunft. Zeigt in I-VI aufeinanderfolgende Entwickelungszustände von der typischen Muskelfaserform bis zur Keulengestalt.

Fig. 7a $\left({ }^{1500 / 1}\right)$. Stück der Querstreifung einer Schwanzmuskelfaser gleicher Dicke wie I aus demselben Präparat.

Fig. $7 \mathrm{~b}(1500 / 1)$. Gleiches Bild aus der Endanschwellung der Faser VI: Ruhestadium. Die Doppelbrechung zwar etwas schwächer als in Fig. 7a, aber doch unvergleichlich stärker als in dem entsprechenden Stadium (Fig. 3a), ja in noch wesentlich früheren Stadien (Fig. 2a) von Raja clavata. 